\title{
Chimpanzees and AIDS research
}

Alfred M. Prince, Jan Moor-Jankowski, Jorg W. Eichberg, Huub Schellekens, Rudolph F. Mauler, Marc Girard and Jane Goodall

Pressure is mounting to relax the regulations on importation of chimpanzees for research. Such a policy is unnecessary and would deepen the plight of an already endangered species.

TWENTY years ago Professor Edward Goldsmith called on scientists not to deplete wild populations of chimpanzees by importing animals for research, but to breed them in captivity instead ${ }^{1.2}$. This viewpoint was subsequently adopted in a joint "Policy Statement on Use of Primates for Biomedical Purposes", issued by the World Health Organization and the European Economic Community in 1982. The statement recommends that chimpanzees and other rare primate species "be considered for use in biomedical research projects only if they are obtained from existing self-sustaining captive breeding colonies". Now there is a new danger to this species, which in the meantime has been so depleted that it is considered threatened in most of its native habitat ${ }^{3.4}$

We have learned of efforts by some in the pharmaceutical industry and by others to persuade governments and WHO that there is a serious shortage of captive chimpanzees for AIDS research. It is alleged that this will impede the development of effective vaccines and therapies to control the disease. The goal is relaxation of the restrictions on importation of chimpanzees from Africa as mandated by the Convention on International Trade in Endangered Species (CITES), to which most Western countries are signatories, as well as a relaxation of the WHO-EEC policy statement on this issue. This was confirmed during a meeting of one of us (J. M-J.) in March of 1988 with the Secretary General of CITES in Lausanne.

\section{Extinction}

We believe that further removal of this species from the wild may lead to its extinction. Moreover, if properly managed, the large number of captive chimpanzees in biomedical laboratories and elsewhere is sufficient for the needs of AIDS researchers.

In a study funded by the US Public Health Service (USPHS), the International Species Inventory System (ISIS) of the International Union for Conservation of Nature and Natural Resources determined that, in the United States as of two years ago, there were about 1,200 chimpanzees in biomedical research laboratories and 400 in zoos 5 . Approximately 350 of these are allocated to breeding programmes and are not available for AIDS research. The availability of captive chimpanzees on the market indicates that, in the United States alone, there are about 1,000 chimpanzees which are not listed by ISIS and which are kept in laboratories or by private breeders, or are kept as pets, photographic models or entertainers; many animals kept by private parties eventually find their way into research laboratories when they become too large and unruly to be easily handled. Additionally, some 100 babies are born annually in private and USPHS-funded chimpanzee breeding centres, and this breeding effort could be considerably enlarged if funds were made available. Outside the United States there are 120 chimpanzees at the Netherlands TNO Primate Centre, 150 at The New York Blood Center's Vilab II at The Liberian Institute for Biomedical Research, and about 200 in research facilities in Japan, many of them involved in breeding.

We estimate, conservatively, that half of the 1,670 chimpanzees currently held in research laboratories could be made available for AIDS research if required.

The main need for the chimpanzee in AIDS research is in the evaluation of potentially protective vaccines. To date the most likely candidate vaccines, based on immunization against the envelope proteins of HIV, have failed to protect in the chimpanzee model ${ }^{6.7}$. Basic research is needed to explain these failures, otherwise a long string of abortive chimpanzee trials can be anticipated. Much of the research now being planned will use the HIV-like simian immunodeficiency virus (SIV) in monkey models. Vaccine development studies in the chimpanzee model can involve assessment of the immunogenicity of candidate vaccines to determine whether these give rise to potentially protective neutralizing antibodies, and to assess the effect of dose and adjuvants. Because most candidate vaccines are derived from recombinant DNA or synthetic peptides, there is little reason why this cannot be done in human volunteers as pioneered in the case of baculovirus-derived gp 160 vaccine by the National Institutes of Health. Such studies do not involve exposure to live virus, so they could also be carried out in chimpanzees otherwise reserved for breeding.

Once optimal doses, adjuvants and immunogens have been identified it is then necessary to determine whether immunization is protective. This is most rapidly and economically done by immunizing two chimpanzees and then challenging them with a small dose of live virus to see if infection is prevented. If it is, clinical trials would ensue.

\section{Hepatitis B}

Relatively large numbers of chimpanzees were employed in the development of the plasma-derived hepatitis B vaccines for two reasons: first, the immunogenicity work was done before the non-infectivity of the products had been established and therefore it could not be carried out in human beings; secondly, as this vaccine was manufactured from virus-containing plasma, lot by lot safety testing in chimpanzees was required. None of these factors applies to potential HIV vaccines made from recombinant-derived or synthetic peptide sources.

If the above strategy is followed, the number of chimpanzees required for AIDS studies will be relatively small and well within the numbers available in biomedical research laboratories.

Relaxation of the restrictions on trade in wild-caught chimpanzees would have a devastating effect on wild populations, virtually all of which are rapidly approaching extinction. If previous practices are allowed to revive, many more chimpanzees of breeding age will be killed in Africa during attempts to capture their infants for export. From discussions with hunters, exporters and importers we estimate that, on average, ten chimpanzees would die for every infant that eventually reached its overseas destination ${ }^{8}$. Such losses surely cannot be justified.

1. Goldsmith, E.I. Nature 219, 1292(1968).

2. Goldsmith, E.I. Science 162, 3858 (1969)

Current Threats to the Survival of Chimpanzees in Equatorial Africa (Jane Goodall Institute, Tucson, Arizona, 1987).

4. African Primate Red Data Book (IUCN Conservation Monitoring Centre, Cambridge, UK, in the press).

Eckholm, E. The New York Times 19 November 1985.

6. Dreesman, G.R. et al. in Retroviruses of Human AIDS and Related Diseases (eds Girard M., DeThe, G. \& Vallette, L. 175-178 (Pasteur Vaccins, Paris 1986)

7. Hu, S.-L. et al. Nature 328, 721-723 (1987)

8. Prince, A.M. in Trends in Bioassay Methodology: In Vivo, In Vitro and Mathematical Approaches 81-97 (US Government Printing Office, 1981).

Alfred $M$. Prince is at the Lindsley F. Kimball Research Institute of the New York Blood Center, 310 East 67 Street, New York, New York 10021, USA, and Vilab II, the Liberian Institute for Biomedical Research, Robertsfield, Liberia. His co-authors are at institutes or laboratories in the United States, the Netherlands, the Federal Republic of Germany and France. 